\title{
Diaphragm fatigue following maximal ventilation in man
}

\author{
C-H. Hamnegård*, S. Wragg**, D. Kyroussis+, G.H. Mills+, M.I. Polkey**, \\ J. Moran+, J. Road ${ }^{+}$, B. Bake ${ }^{++}$, M. Green+, J. Moxham**
}

Diaphragm fatigue following maximal ventilation in man. C-H. Hamnegård, S. Wragg, D. Kyroussis, G.H. Mills, M.I. Polkey, J. Moran, J. Road, B. Bake, M. Green, J. Moxham. (C) ERS Journals Ltd 1996.

ABSTRACT: When highly motivated normal subjects perform maximal isocapnic ventilation, a substantial fall in ventilation is observed during the first minute associated with slowing of the maximum relaxation rate (MRR) of the inspiratory muscles. This suggests that these muscles are excessively loaded, raising the possibility that overt contractile failure of the diaphragm contributes to the fall in ventilation.

We therefore investigated the effect of maximal isocapnic ventilation (MIV) on twitch transdiaphragmatic pressure $(P \mathrm{di}, \mathrm{Tw})$ elicited by cervical magnetic stimulation. We measured $P$ di,Tw before and after $2 \mathrm{~min}$ MIV in nine normal subjects.

Initial mean (SD) ventilation for the nine subjects was $196(15) \mathrm{L}^{\prime} \cdot \mathrm{min}^{-1}$ falling by $35 \%$ at $1 \mathrm{~min}$. Pdi,Tw fell following MIV, at $10 \mathrm{~min}$ was reduced by $24 \%$, and remained substantially reduced 90 min after MIV. No change in $P$ di,Tw was observed during control studies in which subjects were studied with the same protocol but omitting MIV.

We conclude that diaphragmatic contractility is reduced after 2 min maximal isocapnic ventilation and diaphragmatic fatigue may be a limiting factor in maximal ventilation in man.

Eur Respir J., 1996, 9, 241-247.

Diaphragmatic fatigue can be defined as a reduction in the capacity of the muscle to develop force resulting from muscle activity under load that is reversible by rest [1]. Following severe muscular activity, contractility, and in particular the force response to low frequency stimulation, can remain reduced for prolonged periods, [2]. Such low frequency fatigue of the diaphragm has been demonstrated after inspiratory resistive loading [36], following repeated voluntary contractions [7], and after global endurance exercise $[8,9]$.

The ability to sustain maximal isocapnic ventilation (MIV) is an established test of ventilatory capacity [10]. Normal subjects can, initially, achieve ventilation close to $200 \mathrm{~L} \cdot \mathrm{min}^{-1}$, but this declines rapidly during the first $60 \mathrm{~s}$ [10-13]. We have previously shown that during MIV the progressive fall in ventilation and capacity to generate pressure is associated with progressive slowing of the maximum relaxation rate (MRR) of the inspiratory muscles [14, 15], suggesting a peripheral fatiguing process affecting the respiratory muscles. Furthermore, HERSHENSON et al. [16] recently showed that, whereas inspiratory resistive loading preferentially fatigues the rib cage muscles, maximal isocapnic ventilation has a greater effect on performance of the diaphragm. Therefore, it is reasonable to hypothesize that MIV might preferentially load the diaphragm. Slowing of the MRR precedes development of overt contractile failure [17], and evidence that low frequency fatigue is associated with the decline in ventilation during MIV is lacking.
+Respiratory Muscle Laboratory, National Heart and Lung Institute, Brompton Hospital, London, UK. ++Depts of Clinical Physiology and *Pulmonary Medicine, Sahlgrenska University Hospital, Göteborg, Sweden. **Dept of Thoracic Medicine, King's College Hospital, Denmark Hill, London, UK.

Correspondence: C-H. Hamnegård Dept of Pulmonary Medicine Sahlgrenska University Hospital Göteborg

Sweden

Keywords: Diaphragm fatigue magnetic stimulation of the phrenic nerves maximal isocapnic ventilation

Received: May 41995

Accepted after revision October 141995

C-HH was supported by a grant from the Swedish National Association for Heart and Lung Diseases and the University of Göteborg, Sweden.
Low frequency fatigue of the diaphragm can be detected by bilateral electrical stimulation of the phrenic nerves $[3,4]$. However, this technique has disadvantages. Electrical stimulation is often uncomfortable, particularly when attempts are made to achieve supramaximal bilateral stimulation. Furthermore, repeated attempts may be necessary to adequately stimulate the phrenic nerves, and this may lead to twitch potentiation [18] and variability in the transdiaphragmatic pressure response. The technique of cervical magnetic stimulation (CMS) of the phrenic nerve roots has a number of advantages over electrical stimulation $[19,20]$. The stimulation is easily applied, well-tolerated and reproducible, and therefore particularly suitable for the sequential assessment of diaphragmatic contractility.

We therefore measured twitch transdiaphragmatic pressure $(P \mathrm{di}, \mathrm{Tw})$ using CMS of the phrenic nerves, before and after MIV to determine the extent and duration of any diaphragmatic fatigue.

\section{Methods}

Nine male subjects aged $30-49$ yrs were studied. All were familiar with the techniques used. The protocol was approved by the Ethics Committee of the Royal Brompton Hospital and all subjects gave their informed consent. 


\section{Pressure measurements and data processing}

Oesophageal and gastric balloon catheters were positioned and tested in the standard manner [21]. The catheters were connected to differential pressure transducers (Validyne, MP-45-1 $\pm 150 \mathrm{cmH}_{2} \mathrm{O}$ ) and amplified by Validyne carrier amplifiers (Validyne Co., Northridge, CA, USA). These were calibrated before each study with a Universal Pressure Meter (BIO-TEK instruments inc., USA) which was regularly tested for accuracy with a water manometer [22]. All signals were passed into a 12-bit NB-MIO-16 analogue-digital board and a Macintosh Centris 650 computer (Apple Computer, Inc., Cupertino, CA, USA) running LabVIEW ${ }^{\circledR}$ software (National Instruments $(\mathbb{M}$, Austin, TX, USA). All signals were sampled at $100 \mathrm{~Hz}$. Transdiaphragmatic pressure $(P \mathrm{di})$ was defined as the difference between gastric $(P$ ga $)$ and oesophageal $\left(P_{\text {oes }}\right)$ pressures, measured by balloon catheters (PK Morgan, Rainham, Kent, UK).

\section{Maximum isocapnic ventilation}

MIV was performed using the technique described by Mulvey et al. [14]. Subjects breathed through a twoway valve (Hans-Rudolph 2700 series). End-tidal $\mathrm{CO}_{2}$ was monitored by means of a gas monitor (Hewlett-Packard, Waltham, MA, USA). Expiratory flow was measured with a Fleisch No. 4 pneumotachograph head (Fleisch, Lausanne, Switzerland) attached to a brass tube of dimensions 41 by $3.5 \mathrm{~cm}$ to smooth flow, connected to a Mercury CS6 electrospirometer (Mercury Electronics, Glasgow, UK). The electrospirometer computed tidal volume $(V \mathrm{~T})$ and instantaneous minute ventilation (MV) breath-by-breath, and the MV signal was displayed on an oscilloscope screen in front of the subject as visual feedback. Uncalibrated inductance plethysmography (Respitrace, Ambulatory Monitoring Inc., Ardsley NY, USA) was used to detect trends in change of lung volume; rib cage and abdominal bands were positioned at the level of the nipple and just inferior to the umbilicus, respectively.

Inspiratory pressure-time product (PTPinsp) for $P_{\text {oes }}$ and $P$ di, and the expiratory pressure-time product (PTPexp) for $P$ ga, were calculated breath-by-breath. This was accomplished using a custom-designed modification of Labview ${ }^{\circledR} 2.2$ software, which used the flow signal to determine the beginning and end of inspiration and expiration. The areas subtended by the pressure tracings were determined for inspiration and expiration and the mean pressures calculated. The baseline levels for $P$ ga and $P$ di were taken as the resting end-expiratory pressure immediately preceding the MIV, and for Poes was taken as zero pressure, as in the study by BAI et al. [23].

\section{Phrenic nerve stimulation}

The cervical phrenic nerve roots were bilaterally stimulated with CMS using a circular $90 \mathrm{~mm}$ coil (P/N 978400) powered by a Magstim 200 (Magstim Co. Ltd,
Whitland, Dyfed, Wales, UK) [20]. To optimize the position of the coil, the neck was flexed and several stimulations were performed over the spinous processes at varying levels in the midline between $\mathrm{C} 5-\mathrm{C} 7$ until the site of maximum response was located. This spot was marked with an indelible marker and, thereafter, all stimulations were performed at that position and at full stimulator output. Stimulation was performed with subjects wearing a noseclip and with their belt undone.

The pressures elicited by phrenic nerve stimulation ( $P$ oes,Tw, $P$ ga,Tw and $P$ di,Tw) were defined as the difference between baseline pressures immediately before the twitch and the peak pressures following the twitch. Twitches were accepted for analysis when they were performed at functional residual capacity (FRC), as judged both by the end-expiratory oesophageal pressure level and inductance plethysmography and superimposed on a relaxed diaphragm, as judged by the absence of any elevation in $P$ di compared with its baseline level.

\section{Study protocol}

All measurements were made with the subjects seated. On the day prior to the $2 \mathrm{~min}$ MIV, the maximal level of ventilation possible (MLVP) for each subject over a period of $5 \mathrm{~s}$ was determined. Before each $2 \mathrm{~min}$ MIV run, 15 twitches were recorded at relaxed FRC. The stimulations were applied at $30 \mathrm{~s}$ intervals. To avoid twitch potentiation, the subjects were asked to rest for 20 min before each study and were instructed to breathe quietly and remain silent. During each MIV run, the MLVP was set as a target on the oscilloscope screen. The subjects were vigorously encourage to achieve this target throughout the run. No pattern of tidal volume $(V \mathrm{~T})$, respiratory duty cycle $(t \mathrm{I} / t \mathrm{TOT})$ or respiratory rate was imposed on the subjects. At 5 min post-MIV, 10 twitches were performed at relaxed FRC. These were repeated at 10, 20 40, 60 and 90 min. During control studies, subjects were studied with the same protocol but omitting MIV.

\section{Statistical analysis}

Statistical analysis was performed using StatView 4.0 (Abacus concepts, Inc., Berkley, CA, USA). Changes in the group mean $P$ di,Tw before and $10 \mathrm{~min}$ following MIV were compared using a paired t-test. The result are expressed as the mean and SD unless otherwise indicated. A p-value of less than 0.05 was considered statistically significant.

\section{Results}

\section{Two minute MIV run}

Data from the run are summarized in table 1 with traces from a representative subject in figure 1. Ventilation 
Table 1. - Absolute values of ventilation, pressure-time product during inspiration (PTPinsp) and pressure-time product during expiration (PTPexp) during 2 min MIV run

\begin{tabular}{lcccc}
\hline Sub. & MV & PTPinsp,Pdi & PTPinsp,Poes & PTPexp,Pga \\
No. & L $\cdot$ min $^{-1}$ & $\mathrm{kPa} \cdot \mathrm{s} \cdot \mathrm{min}^{-1}$ & $\mathrm{kPa} \cdot \mathrm{s} \cdot \mathrm{min}^{-1}$ & $\mathrm{kPa} \cdot \mathrm{s} \cdot \mathrm{min}^{-1}$
\end{tabular}

\begin{tabular}{lccrl}
\hline \multicolumn{5}{l}{ Within initial five seconds } \\
1 & 172 & 164 & 142 & \\
2 & 192 & 193 & 175 & 295 \\
3 & 211 & 189 & 152 & 312 \\
4 & 210 & 172 & 153 & 423 \\
5 & 195 & 139 & 81 & 215 \\
6 & 199 & 158 & 150 & 304 \\
7 & 193 & 177 & 163 & 268 \\
8 & 178 & 227 & 174 & 341 \\
9 & 215 & 169 & 130 & 298 \\
\hline \multirow{2}{*}{ Mean } & 196 & 177 & 147 & 304 \\
SD & 15 & 25 & 29 & 57 \\
\hline
\end{tabular}

\begin{tabular}{lccrr}
\hline \multicolumn{5}{l}{ At plateau level of ventilation } \\
1 & 149 & 87 & 76 & 121 \\
2 & 109 & 94 & 73 & 157 \\
3 & 148 & 94 & 85 & 123 \\
4 & 105 & 82 & 78 & 93 \\
5 & 139 & 101 & 58 & 99 \\
6 & 134 & 95 & 102 & 132 \\
7 & 102 & 81 & 71 & 145 \\
8 & 115 & 101 & 107 & 142 \\
9 & 149 & 82 & 78 & 91 \\
\hline \multirow{2}{*}{ Mean } & 128 & 91 & 81 & 123 \\
SD & 20 & 8 & 15 & 24 \\
\hline
\end{tabular}

MIV: maximal isocapnic ventilation; MV: minute ventilation; $P$ di: transdiaphragmatic pressure; $P_{\text {oes: }}$ oesophageal pressure; $P$ ga: gastric pressure; Sub.: subject. declined rapidly from the beginning of the run in all subjects (fig. 2), reaching a plateau after $60 \mathrm{~s}$. Initial mean (SD) MIV was $196(15) \mathrm{L} \cdot \mathrm{min}^{-1}$, falling by a mean of $37 \%$ at 2 min. Parallel with the decline in ventilation there was a fall in the pressure-time product (PTP) generated both by the inspiratory and expiratory muscles (fig. 3). Initial mean PTPinsp,Pdi (SD) was 177 (25) $\mathrm{kPa} \cdot \mathrm{s} \cdot \mathrm{min}^{-1}$ and fell by $48 \%$ at the plateau. Similarly PTPinsp,Poes was 147 (29) $\mathrm{kPa} \cdot \mathrm{s} \cdot \mathrm{min}^{-1}$ and fell by $45 \%$, and initial PTPex,Pga was $304(57) \mathrm{kPa} \cdot \mathrm{s} \cdot \mathrm{min}^{-1}$ and fell by $60 \%$. The respiratory duty cycle, $T \mathrm{I} / T$ tot (SD), was $0.48(0.03)$ at the start and $0.52(0.05)$ at the end of the run. To estimate the relative contribution from respiratory muscles, the slope of $P$ ga/ $P$ oes was determined, where

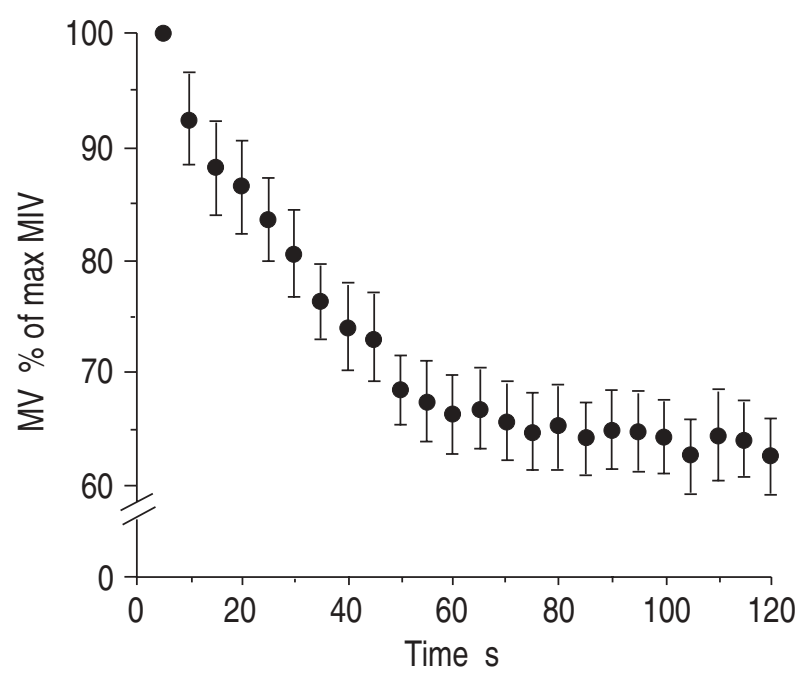

Fig. 2. - Minute ventilation (MV) (mean and SEM) during 2 min maximal isocapnic ventilation (MIV) in nine normal subjects.
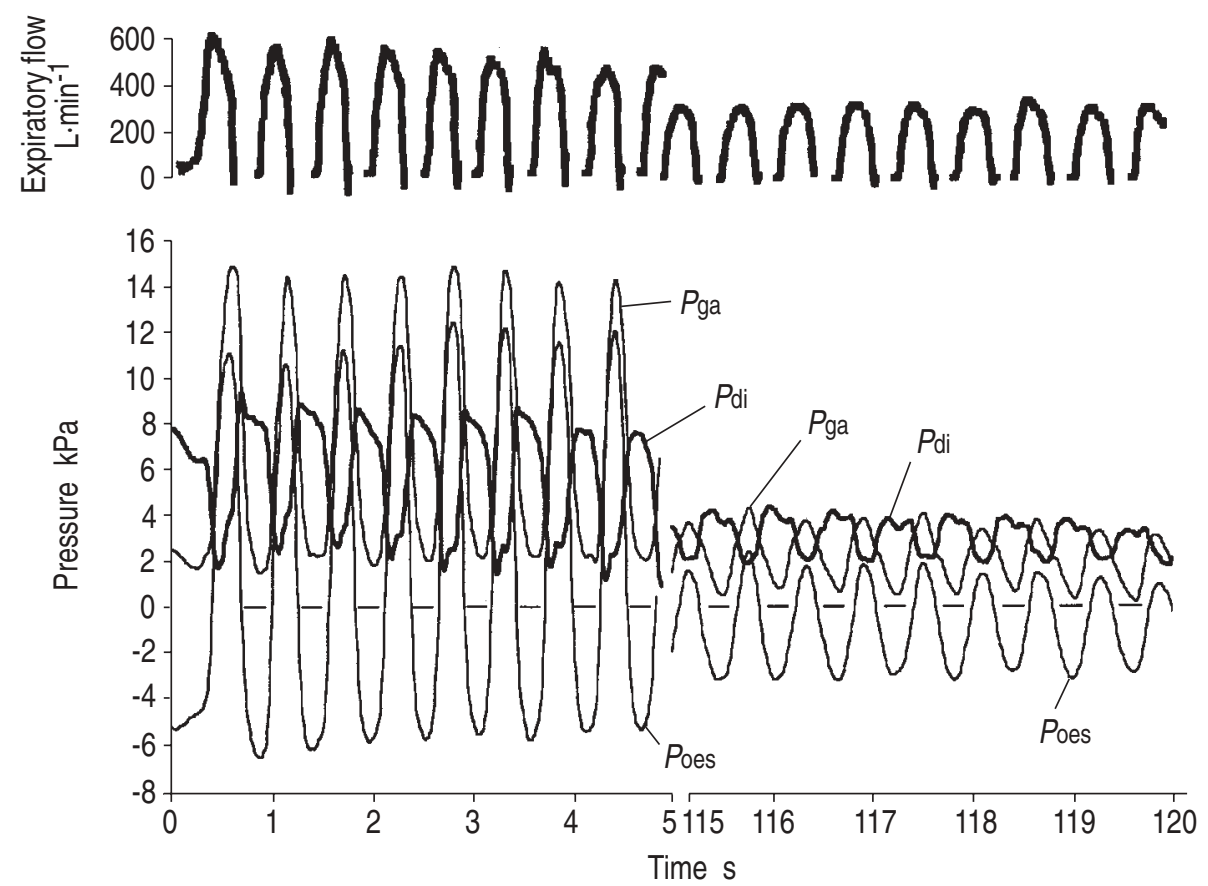

Fig. 1. - Pressure and flow traces obtained during the first 5 and final $5 \mathrm{~s}$ of maximal isocapnic ventilation (MIV) in a representative subject. $P$ ga: gastric pressure; $P_{\text {di: }}$ transdiaphragmatic pressure (thick line); $P_{\text {oes: }}$ oesophageal pressure. 


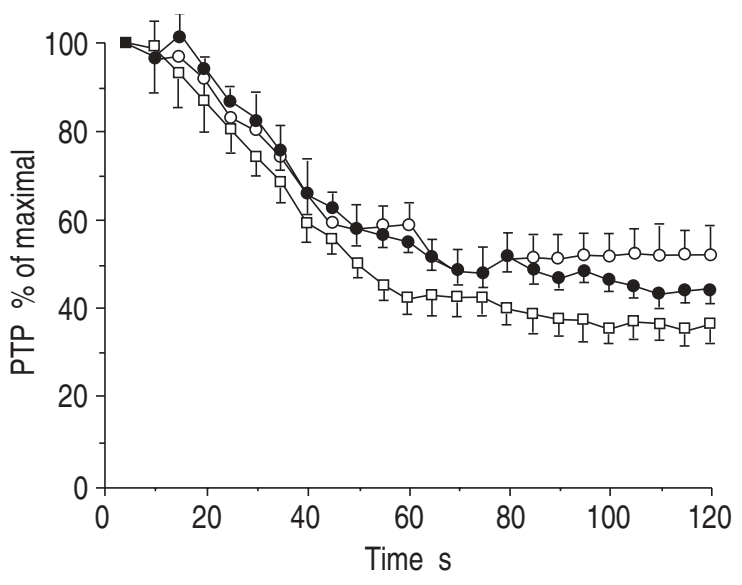

Fig. 3. - Mean (SEM) inspiratory (insp) and expiratory (exp) pressuretime products (PTP) during 2 min maximal isocapnic ventilation (MIV) in nine normal subjects. - $\longrightarrow$ : PTPinsp, Poes; $\longrightarrow-$ : PTPinsp, $P$ di; $-\_-$PTPexp,Pga. For further definitions see legend to table 1.

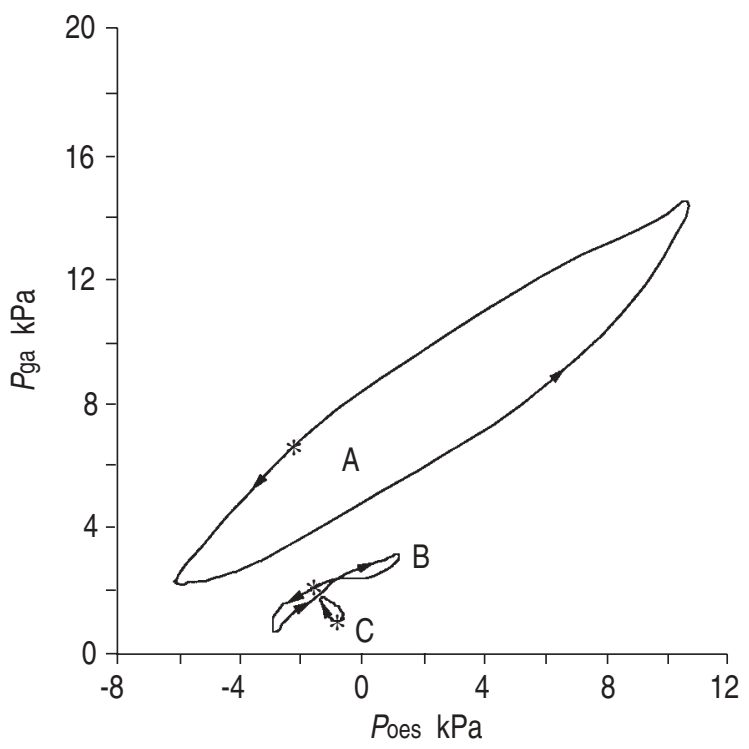

Fig. 4. - Representative single-breath plots of gastric pressure $(P$ ga $)$ against oesophageal pressure $\left(P_{\mathrm{oes}}\right)$ illustrating the pattern of respiratory muscle recruitment. A: at the start of MIV showing predominance of the rib cage accessory and abdominal muscles; B: at the end of maximal isocapnic ventilation (MIV), little change in the pattern of recruitment is observed; $\mathrm{C}$ : by contrast, during tidal breathing there is diaphragmatic predominance. a negative slope reflects predominance of expiratory abdominal and inspiratory chest wall accessory muscles (fig. 4). For all subjects there was a predominance of activity in these muscles with little or no change throughout the MIV run.

\section{Twitch pressures}

$P$ di,Tw was significantly decreased following MIV in all subjects (table 2 and fig. 5). For the group, mean $P$ di,Tw (SD) fell from 3.23 (0.68) kPa before MIV to 2.47 (0.58) $\mathrm{kPa} 10 \mathrm{~min}$ after MIV, a mean fall (SD) of 23 (10)\% (p<0.01). Poes,Tw (SD) and Pga,Tw (SD) fell from $1.93(0.54)$ and $1.24(0.53) \mathrm{kPa}$ before MIV to $1.48(0.42)$ and $0.92(0.46) \mathrm{kPa} 10 \mathrm{~min}$ after MIV $(\mathrm{p}<0.01)$. Mean $P$ di,Tw remained substantially reduced, to $83 \%$ of baseline, at $90 \mathrm{~min}$ after MIV (fig. 6). There was no change in the ratio Poes,Tw:Pga,Tw, before and after MIV.

During control studies, subjects were studied with the same protocol but omitting MIV. Pdi,Tw, $P_{\text {oes,Tw }}$ and $P$ ga,Tw did not fall during the control run (fig. 6).

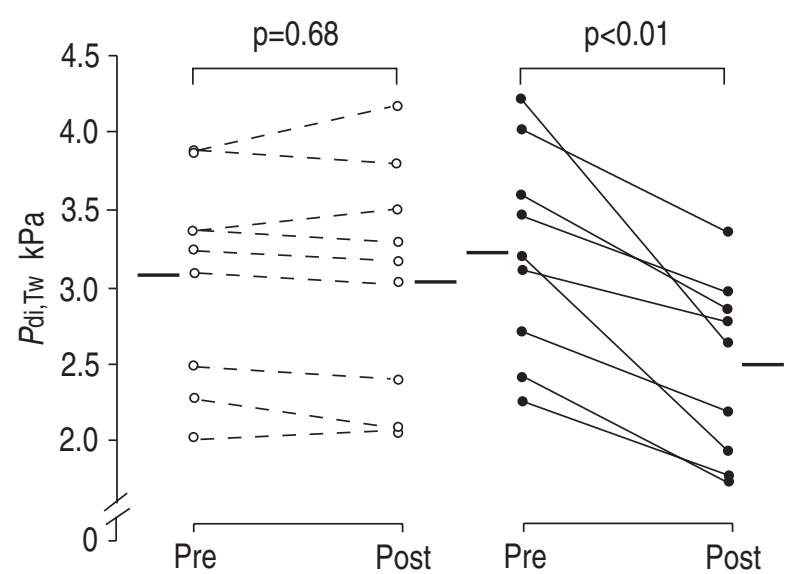

Fig. 5. - Mean twitch transdiaphragmatic pressure ( $\left.P_{\mathrm{di}, T w}\right)$ (thick bar) for each subject $(\mathrm{n}=9)$ before and $10 \mathrm{~min}$ after maximal isocapnic ventilation (MIV) (closed circles) and control run (open circles). There is a statistically significant fall in $P_{\mathrm{di}, \mathrm{Tw}}(\mathrm{p}<0.01)$ after MIV but not after the control run.

Table 2. - Characteristics of the subjects studied, functional parameters and twitch transdiaphragmatic pressure $(P \mathrm{di}, \mathrm{Tw})$ before and $10 \mathrm{~min}$ after MIV

\begin{tabular}{|c|c|c|c|c|c|c|c|}
\hline \multirow[b]{2}{*}{$\begin{array}{l}\text { Sub. } \\
\text { No. }\end{array}$} & \multirow[b]{2}{*}{$\begin{array}{l}\text { Age } \\
\text { yrs }\end{array}$} & \multirow[b]{2}{*}{$\begin{array}{l}\text { Height } \\
\mathrm{cm}\end{array}$} & \multirow[b]{2}{*}{$\begin{array}{c}\text { Weight } \\
\mathrm{kg}\end{array}$} & \multirow[b]{2}{*}{$\begin{array}{c}\mathrm{VC} \\
\mathrm{L}\end{array}$} & \multirow{2}{*}{$\begin{array}{c}\text { Sniff } \\
P \text { di,max } \\
\mathrm{kPa}\end{array}$} & \multicolumn{2}{|c|}{$P$ di,Tw } \\
\hline & & & & & & $\begin{array}{c}\text { Before MIV } \\
\mathrm{kPa}\end{array}$ & $\begin{array}{c}\text { After MIV } \\
\mathrm{kPa}\end{array}$ \\
\hline 1 & 38 & 179 & 76 & 6.6 & 13.9 & 4.23 & 2.63 \\
\hline 2 & 49 & 176 & 72 & 6.2 & 15.3 & 3.2 & 1.93 \\
\hline 3 & 42 & 186 & 85 & 6.8 & 14.8 & 2.27 & 1.77 \\
\hline 4 & 38 & 181 & 68 & 6.2 & 14.6 & 2.42 & 1.74 \\
\hline 5 & 43 & 182 & 79 & 5.7 & 16.3 & 3.48 & 2.97 \\
\hline 6 & 30 & 192 & 82 & 6.8 & 13.6 & 2.72 & 2.19 \\
\hline 7 & 32 & 175 & 80 & 6.6 & 15.1 & 3.12 & 2.78 \\
\hline 8 & 33 & 198 & 95 & 5.9 & 16 & 4.03 & 3.36 \\
\hline 9 & 39 & 186 & 82 & 6.5 & 15.1 & 3.61 & 2.85 \\
\hline Mean & 38 & 184 & 80 & 6.4 & 15.0 & 3.23 & 2.47 \\
\hline SD & 6 & 8 & 8 & 0.4 & 0.9 & 0.68 & 0.58 \\
\hline
\end{tabular}

VC: vital capacity; Pdi,max: maximum transdiaphragmatic pressure; MIV: maximal isocapnic ventilation; Sub.: subject. 


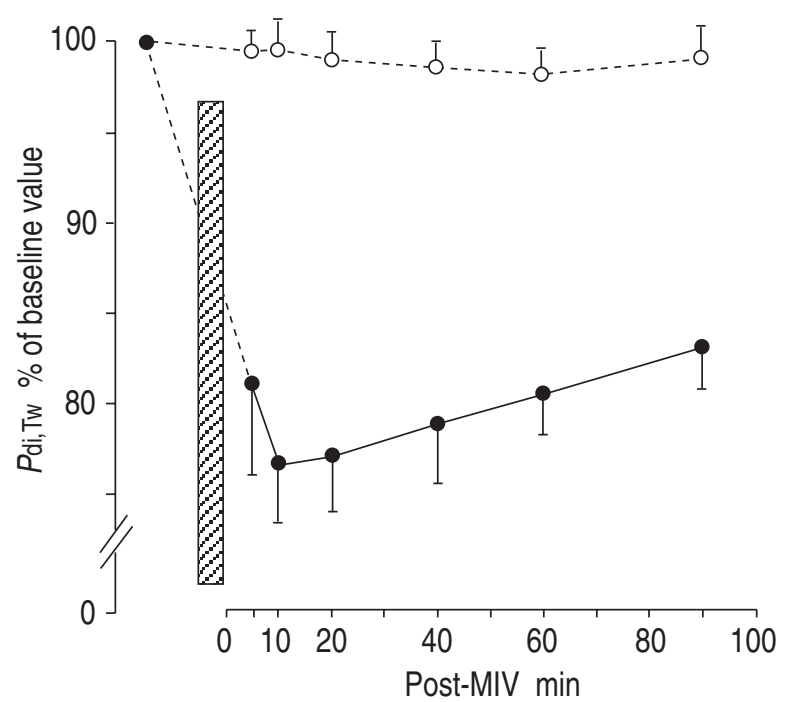

Fig. 6. - Fall and recovery of mean (and SEM) twitch transdiaphragmatic pressure $(P \mathrm{di}, \mathrm{Tw})$ after 2 min maximal isocapnic ventilation (MIV, hatched column) (closed symbols) and control run (open symbols). After MIV, $P$ di,Tw was reduced by $24 \%$ and was still reduced by $17 \%$ at $90 \mathrm{~min}$.

\section{Discussion}

The main finding of this study is that the fall in minute ventilation which occurs during MIV is associated with a parallel fall in the pressure-time product both by inspiratory and expiratory muscles. This, in turn, is associated with a fall and subsequent slow recovery of $P$ di,Tw typical of low frequency fatigue. These observations suggest that low frequency diaphragm fatigue is, at least partially, responsible for the decline in performance observed in MIV. Before discussing these observations, some methodological issues will be addressed.

\section{Supramaximality}

WRAGG and co-workers [20] and SimiLOvski et al. [19] have previously shown that CMS can achieve a supramaximal stimulation. Five of the subjects in the present study were participants in the study of WRAGG and coworkers [20], and in these supramaximality of stimulation had been demonstrated as judged by a plateau of $P$ di,Tw and (in three subjects) diaphragm electromagnetic activity (EMG) in response to increasing intensity of magnetic stimulation [20]. However low frequency fatigue (LFF) of the diaphragm can be detected using submaximal stimulation [4]. Moreover, the reproducibility of $P \mathrm{di}, \mathrm{Tw}$ in the fresh state (within occasion coefficient of variation less than $5 \%$ ) and the lack of a fall of $P$ di,Tw after the control run suggests that the observed fall in $P$ di,Tw after MIV is not due to the stimulation technique.

\section{Specificity of cervical magnetic stimulation}

During CMS, spread of the magnetic field causes activation of muscles acting on the upper rib cage, as well as the diaphragm [19]. It could, thus, be argued that a change in $P$ di,Tw is due to alteration of the contractile properties of the extradiaphragmatic muscles. However, stimulation of the muscles of the upper thorax, whether by magnetic [24] or electrical stimulation [25], does not produce a significant transdiaphragmatic pressure. Furthermore, the ratio $P_{\mathrm{oes}}, \mathrm{Tw}: P_{\mathrm{ga}}, \mathrm{Tw}$, did not change after MIV, which suggests no important alteration of the pattern of muscle contraction in response to CMS. It is, thus, likely that activation of extradiaphragmatic muscles merely allows greater efficiency of diaphragmatic contraction, and that the fall in Pdi,Tw observed represents a genuine change in diaphragmatic contractility.

\section{Influence of lung volume}

$P$ di,Tw has a linear inverse relationship with lung volume [26]. In the present study, lung volume was not measured directly but $P$ oes and $P$ di traces were monitored prior to the twitch to ensure that lung volume was similar before and after MIV, and also inductive plethysmography was used. Moreover, Mulvey et al. [14], using an identical MIV technique, found no change in endexpiratory lung volume.

\section{Changes in abdominal compliance}

Alterations in abdominal compliance could affect $P$ di,Tw. However, fatigue does not alter the passive length-tension characteristics of muscle [27], and we confirmed relaxation of the abdominal muscles by monitoring $P$ ga. Moreover, in our study $P$ oes,Tw and $P$ ga,Tw were reduced by the same proportion, which would not be the case if abdominal compliance was reduced. Thus, we can be reasonably confident that there was no substantial change in abdominal compliance after MIV.

\section{Twitch potentiation}

To avoid twitch potentiation of the diaphragm, the subjects were asked to rest for 20 min before measurements. This period was considered appropriate because we have previously shown that the effects of twitch potentiation are measurable up to 20 min after a maximal voluntary contraction of the diaphragm, though this effect is of negligible importance after $10 \mathrm{~min}$ [18]. This phenomenon probably explains the observation that the reduction in $P$ di,Tw was greatest at 10 and not at 5 min after MIV, since twitch potentiation after the MIV could be expected to offset the reduction in $P$ di,Tw amplitude caused by fatigue.

\section{Changes in pressure-time product and ventilation}

The relationship between pressures, ventilation and pressure-time products observed in the present study are comparable to those reported previously [23, 28, 29]. However, data documenting the decline in pressure-time product during the course of a 2 min MIV have not previously been presented. The inspiratory pressure-time 
products for transdiaphragmatic pressure (PTP,Pdi) and oesophageal pressure (PTP,Poes) declined rapidly during the first minute of MIV to approximately 48 and $45 \%$ of baseline values, respectively. By contrast, the fall in expiratory pressure-time product (PTP,Pga), which could be considered to represent expiratory muscle activity, was significantly greater $(60 \%)$. Thus, it is possible that reduction in pressure generation by the expiratory muscles makes an important contribution to the decline in ventilation. In this context, it is of interest that data showing fatigue of these muscles during MIV has recently been reported $[30,31]$. During MIV, expiratory $P$ ga substantially exceeds the pressure required to reach maximal expiratory flow. The excessive $P$ ga in expiration during isocapnic hyperpnoea might reflect an undesirable overriding of the normal control mechanisms that operate during exercise in normal subjects, where expiratory pressure rarely exceeds that necessary to generate maximal flow [23, 32, 33]. Alternatively, vigorous abdominal muscle contraction during expiration might be of functional importance by displacing the diaphragm cranially. This would store elastic and gravitational energy, which could be recovered in the subsequent inspiration. A further benefit of lung compression would be an increase in diaphragm length, and consequently in diaphragm force-generating capacity.

\section{Diaphragmatic fatigue}

A reduction in $P$ di,Tw after loading is evidence of peripheral low frequency fatigue of the diaphragm [6]. In this study, $P$ di,Tw fell to $77 \%$ of the control value $10 \mathrm{~min}$ after MIV and was still reduced to $83 \%$ at 90 min postMIV; this pattern of slow recovery is typical of low frequency fatigue [2].

No studies have looked specifically at the effect of hyperpnoea on $P$ di,Tw but high levels of ventilation have been achieved in studies of whole-body exercise. JOHNSON et al. [8] described subjects who were able to achieve a maximum ventilation of $157 \mathrm{~L} \cdot \mathrm{min}^{-1}$. They found a reduction in $P$ di,Tw in nine of 11 subjects, with almost complete recovery $70 \mathrm{~min}$ after exercise. The subjects described by MADOR et al. [9] achieved a maximal ventilation of $90 \mathrm{~L} \cdot \mathrm{min}^{-1}$; they found a fall in $P$ di,Tw in seven of 10 subjects, with a partial recovery 60 min after exercise. In the present study, ventilatory muscles were stressed in isolation, thus confirming that activity of the respiratory muscles alone can, in some circumstances, produce diaphragmatic fatigue. We have also shown a greater and more sustained degree of fatigue than has been previously documented and this is presumably due to the greater ventilation achieved during MIV than wholebody exercise.

Low frequency fatigue has been demonstrated in a wide variety of skeletal muscles, including the diaphragm, but there are no data documenting the occurrence of LFF of the respiratory muscles in clinical situations. Slowing of the MRR has previously been suggested as evidence of fatigue in clinical and physiological studies [14, $15,17,34]$. However, slowing of the MRR is a normal adaptive mechanism to preserve force-generating capacity of the diaphragm [35] in response to a presumed slowing of phrenic nerve firing rate; the theory of "muscular wisdom" [36]. However, slowing of MRR precedes task failure [17] and is not evidence of LFF. The present study is of importance because it confirms the occurrence of LFF after MIV.

CMS is technically easier to use and more acceptable to patients than bilateral transcutaneous electrical stimulation [37]. Since LFF of the diaphragm can be detected using the $P$ di,Tw elicited by CMS, it is likely that the technique will be of value for investigating the occurrence of LFF in clinical studies, and one such study has been reported [38].

We conclude that cervical magnetic stimulation of the phrenic nerves allows reproducible sequential assessment of diaphragm contractility. Following 2 min maximal isocapnic ventilation diaphragm contractility is reduced. This reduction of twitch transdiaphragmatic pressure is long-lasting. It is possible that diaphragm fatigue is an important factor limiting maximal ventilation in man, and it is likely that the technique of cervical magnetic stimulation can be used to investigate the role of diaphragmatic fatigue in ventilatory failure in physiological studies of patients.

\section{References}

1. NHLBI Workshop. Respiratory muscle fatigue. Report of the Respiratory Muscle Fatigue Workshop Group. Am Rev Respir Dis 1990; 142: 474-480.

2. Edwards RHT, Hill DK, Jones DA, Merton PA. Fatigue of long duration in human skeletal muscle after exercise. J Physiol Lond 1977; 272: 769-778.

3. Aubier M, Farkas G, De Troyer A, Mozes R, Roussos C. Detection of diaphragmatic fatigue in man by phrenic stimulation. J Appl Physiol: Respirat Environ Exercise Physiol 1981; 50: 538-544.

4. Moxham J, Morris AJR, Spiro SG, Edwards RHT, Green M. Contractile properties and fatigue of the diaphragm in man. Thorax 1981; 36: 164-168.

5. Moxham J, Edwards RHT, Aubier M, et al. Changes in EMG power spectrum (high-to-low ratio) with force fatigue in humans. J Appl Physiol: Respirat Environ Exercise Physiol 1982; 53: 1094-1099.

6. Aubier M, Murciano D, Lecocgui Y, Viires N, Pariente R. Bilateral phrenic stimulation: a simple technique to assess diaphragmatic fatigue in humans. J Appl Physiol 1985; 58: 58-64.

7. Bellemare F, Bigland-Ritchie B. Central components of diaphragmatic fatigue assessed by phrenic nerve stimulation. J Appl Physiol 1987; 62: 1307-1316.

8. Johnson BD, Babcock MA, Suman OE, Dempsey JA. Exercise-induced diaphragmatic fatigue in healthy humans. J Physiol Lond 1993; 460: 385-405.

9. Mador MJ, Magalang UJ, Rodis A, Kufel TJ. Diaphragmatic fatigue after exercise in healthy human subjects. Am Rev Respir Dis 1993; 148: 1571-1575.

10. Gandevia B, Hugh-Jones P. Terminology for measurements of ventilatory capacity. Thorax 1957; 12: 290293.

11. Freedman S. Sustained maximum voluntary ventilation. Respir Physiol 1970; 41: 230-244. 
12. Leith DE, Bradley M. Ventilatory muscle strength and endurance training. J Appl Physiol 1976; 41: 508-516.

13. Tenney SM, Reese RE. The ability to sustain great breathing efforts. Respir Physiol 1968; 5: 187-201.

14. Mulvey DA, Koulouris NG, Elliott MW, Laroche CM, Moxham J, Green M. Inspiratory muscle relaxation rate after voluntary maximal isocapnic ventilation in humans. J Appl Physiol 1991; 70: 2173-2180.

15. Kyroussis D, Mills G, Hamnegård C-H, et al. Inspiratory muscle relaxation rate assessed from sniff nasal pressure. Thorax 1994; 49: 1127-1133.

16. Hershenson MB, Kikuchi Y, Tzelepis GE, McCool FD. Preferential fatigue of the rib cage muscles during inspiratory resistive loaded ventilation. J Appl Physiol 1989; 66: 750-754.

17. Esau SA, Bellemare F, Grassino A, Permutt S, Roussos C, Pardy RL. Changes in relaxation rate with diaphragmatic fatigue in humans. J Appl Physiol: Respirat Environ Exercise Physiol 1983; 54: 1353-1360.

18. Wragg S, Hamnegård C, Road J, Kyroussis D, Green M, Moxham J. Potentiation of diaphragmatic twitch after voluntary contraction in normal subjects. Thorax 1994; 49: $1234-1237$

19. Similovski T, Fleury B, Launois S, Cathala HP, Bouche P, Durenne JP. Cervical magnetic stimulation: a new painless method for bilateral phrenic stimulation in conscious humans. J Appl Physiol 1989; 67: 1311-1318.

20. Wragg S, Aquilina R, Moran J, et al. Comparison of cervical magnetic stimulation and bilateral percutaneous electrical stimulation of the phrenic nerves in normal subjects. Eur Respir J 1994; 7: 1788-1792.

21. Baydur A, Pangiotis K, Behrakis K, Zin WA, Jaeger M, Milic-Emili JA. A simple method of assessing the validity of the esophageal balloon technique. Am Rev Respir Dis 1982; 126: 788-791.

22. Sixt R, Bake B. A simple pressure calibrator. Scand J Clin Lab Invest 1976; 36: 1-2.

23. Bai TR, Rabinovitch BJ, Pardy RL. Near-maximal voluntary hyperpnea and ventilatory muscle function. $J \mathrm{Appl}$ Physiol: Respirat Environ Exercise Physiol 1984; 57: 1742-1748.

24. Mills G, Kyroussis D, Hamnegård C-H, Wragg S, Moxham $\mathrm{J}$, Green $\mathrm{M}$. Chest wall activation during cervical magnetic phrenic stimulation (CMPS) does not produce inspiratory pressures. Am J Respir Crit Care Med 1995; 151: A414.

25. Similowski T, Yan S, Gauthier AP, Macklem PT, Bellemare F. Contractile properties of the human diaphragm during chronic hyperinflation. N Engl J Med 1991; 325: 917-923.

26. Hamnegård C, Wragg S, Mills G, et al. The effect of lung volume on transdiaphragmatic pressure. Eur Respir $J$ 1995; 8: 1532-1536.

27. Ralston HJ, Inman VT, Strait LA, Shaffrath MD. Mechanics of human isolated voluntary muscle. Am J Physiol 1947; 151: 612-620.

28. Milic-Emili J, Orzalesi MM, Cook CD, Turner JM. Respiratory thoracoabdominal mechanics in man. J Appl Physiol 1964; 19: 217-223.

29. Ogilvie CM, Stone RW, Marshall R. The mechanics of breathing during the maximum breathing capacity test. Clin Sci 1955; 14: 101-107.

30. Kyroussis D, Mills G, Polkey MI, Hamnegård C-H, Moxham J, Green M. Magnetic stimulation of the abdominal muscles. Am J Respir Crit Care Med 1995; 151: A415.

31. Kyroussis D, Mills G, Hamnegård C-H, et al. Maximum relaxation rate of the expiratory muscles slows with maximum isocapnic ventilation. Eur Respir J 1994; 7 : 286s.

32. Olafsson S, Hyatt RE. Ventilatory mechanics and expiratory flow limitation during exercise in normal subjects. J Clin Invest 1969; 48: 564-573.

33. Hesser CM, Linnarsson D, Fagraeus L. Pulmonary mechanics and work of breathing at maximal ventilation and raised air pressure. J Appl Physiol: Respirat Environ Exercise Physiol 1981; 50: 747-753.

34. Goldstone J, Green M, Moxham J. Maximum relaxation rate of the diaphragm during weaning from mechanical ventilation. Thorax 1994; 49: 54-60.

35. Enoka RM, Stuart DG. Neurobiology of muscle fatigue. J Appl Physiol 1992; 72: 1631-1648.

36. Marsden C, Meadows J. Muscular wisdom that minimized fatigue during prolonged effort in man: peak rates of motoneuron discharge and slowing of discharge during fatigue. In: Desmedt J, ed. Motor Control Mechanisms in Health and Disease. New York; Raven Press, 1983; pp. 169-211.

37. Wragg S, Hamnegård C-H, Kyroussis D, Mills G, Green M, Moxham J. Assessment of diaphragm strength in patients using cervical magnetic stimulation. Am J Respir Crit Care Med 1994; 149: A130.

38. Polkey MI, Kyroussis D, Keilty SEJ, et al. Exhaustive treadmill exercise does not reduce twitch transdiaphragmatic pressure in patients with COPD. Am J Respir Crit Care Med 1995; 151: 959-964. 